\title{
A novel approach to inhibit HIV-I infection and enhance lysis of HIV by a targeted activator of complement
} Yuanyong Xu ${ }^{\dagger 1}$, Chuanfu Zhang ${ }^{\dagger 1}$, Leili Jia ${ }^{\dagger 1}$, Cuirong Wen², Huihui Liu ${ }^{3}$, Yong Wang ${ }^{1}$, Yansong Sun ${ }^{1}$, Liuyu Huang ${ }^{1}$, Yusen Zhou*4 and Hongbin Song*1

Address: ${ }^{1}$ Institute of Disease Control and Prevention, Academy of Military Medical Science, Beijing 100071, PR China, ${ }^{2302}$ Hospital of People's Liberation Army, Beijing 100039, PR China, ${ }^{3}$ Chinese Center for Disease Control and Prevention, Department of Epidemiology, Beijing 100050 , PR China and ${ }^{4}$ State Key Laboratory of Pathogen and Biosecurity, Beijing Institute of Microbiology and Epidemiology, Beijing 100071, PR China

Email: Yuanyong Xu - xyy_827@sina.com; Chuanfu Zhang - hnzcf@126.com; Leili Jia - jialeili@163.com;

Cuirong Wen - wen_cuirong@126.com; Huihui Liu - liuhuihui323@sina.com; Yong Wang - ywang7508@yahoo.com.cn;

Yansong Sun - sunys1964@hotmail.com; Liuyu Huang - huangly@nic.bmi.ac.cn; Yusen Zhou* - yszhou@nic.bmi.ac.cn;

Hongbin Song* - hongbinsong@263.net

* Corresponding authors †Equal contributors

Published: 12 August 2009

Virology Journal 2009, 6:123 doi:10.1186/1743-422X-6-123
Received: I June 2009

Accepted: 12 August 2009

This article is available from: http://www.virologyj.com/content/6/I/I23

(c) 2009 Xu et al; licensee BioMed Central Ltd.

This is an Open Access article distributed under the terms of the Creative Commons Attribution License (http://creativecommons.org/licenses/by/2.0), which permits unrestricted use, distribution, and reproduction in any medium, provided the original work is properly cited.

\footnotetext{
Abstract

Background: The complement system is one of the most potent weapons of innate immunity. It is not only a mechanism for direct protection against invading pathogens but it also interacts with the adaptive immunity to optimize the pathogen-specific humoral and cellular defense cascades in the body. Complement-mediated lysis of HIV is inefficient but the presence of HIV particles results in complement activation by the generation of many $\mathrm{C} 3$-fragments, such as $\mathrm{C} 3 \mathrm{dg}$ and $\mathrm{C} 3 \mathrm{~d}$. It has been demonstrated that activation of complement can enhance HIV infection through the binding of special complement receptor type 2 expression on the surface of mature $B$ cells and follicular dendritic cells.

Presentation of the hypothesis: Previous studies have proven that the complement-mediated antibody-dependent enhancement of HIV infection is mediated by the association of complement receptor type 2 bound to the $\mathrm{C} 3$ fragment and deposited on the surface of HIV virions. Thus, we hypothesize that a new activator of complement, consisting of a target domain (C3-binding region of complement receptor type 2) linked to a complement-activating human IgGI Fc domain (CR2-Fc), can target and amplify complement deposition on HIV virions and enhance the efficiency of HIV lysis.

Testing the hypothesis: Our hypothesis was tested using cell-free HIV-I virions cultivated in vitro and assessment of virus opsonization was performed by incubating appropriate dilutions of virus with medium containing normal human serum and purified CR2-Fc proteins. As a control group, viruses were incubated with normal human serum under the same conditions. Virus neutralization assays were used to estimate the degree of CR2-Fc-enhanced lysis of HIV compared to untreated virus.

Implications of the hypothesis: The targeted complement activator, CR2-Fc, can be used as a novel approach to HIV therapy by abrogating the complement-enhanced HIV infection of cells.
} 


\section{Background}

The human immunodeficiency virus (HIV) causes severe immune deficiency in humans and currently affects up to 42 million people worldwide. To date, there are no effective vaccines against HIV infection due to a number of issues. Firstly, there have been several recent failures of potential vaccine candidates in clinical trials. In 2003, two phase 3 trials using gp120 protein for vaccination that were aimed to raise sterilizing, antibody-mediated immunity, failed to protect vaccinees from HIV infection $[1,2]$. Another vaccine trial using a different strategy (V520 of Merck) was stopped prematurely in September 2007 due to evidence that vaccinees may have been more susceptible to HIV infection than placebo control individuals [3]. Secondly, no effective therapeutic approach for "curing" HIV infected individuals is currently under clinical investigation. Current therapies for HIV infection using highly active antiretroviral therapy (HAART) are not able to eliminate virus completely and complications of these therapies include severe side effects and viral resistance that may establish latent reservoirs of HIV.

The complement system is a key component of innate immunity and provides a first line of defense against invading pathogens that can bridge the innate and adaptive arms of the immune system $[4,5]$. It is not only a mechanism for direct protection against invading pathogens but also interacts with the adaptive immune system to optimize the pathogen-specific humoral and cellular defense cascade in the body, especially for viral pathogens. HIV, however, has evolved several mechanisms to evade complement-mediated lysis (CML) and exploit the complement system to increase viral infectivity [6]. Thus, in light of recent failures for vaccine design, the present study proposes an innovative approach to find a novel targeted activator of complement for the elimination of HIV.

\section{Presentation of the hypothesis}

\section{Interaction of HIV with the complement system}

HIV infection leads to the immediate activation of the complement system, even in the absence of HIV-specific antibodies. However, after seroconversion, the presence of HIV-specific antibodies triggers further activation of the classical complement pathway [7]. Antibodies that may enhance HIV infection in vitro were described shortly after HIV had first been isolated. Robinson et al. [8] found that sera from HIV-infected individuals enhance in vitro HIV infection of the complement receptor type 2 (CR2; CD21)-bearing T lymphoblastoid cell line, MT2. The same authors demonstrated that this enhancement was dependent on antibodies and mediated by complement and coined the term complement-mediated antibodydependent enhancement (C-ADE) [9]. The mechanism of $\mathrm{C}$-ADE has been investigated by several studies during the past two decades. As summarized by Robinson et al. [8], binding of antibody to gp4 1 initiates the complement cascade and leads to the deposition of the C3dg complement component on the virion. Opsonized viruses subsequently bind to CR2 distributed on mature B cells and follicular dendritic cells (FDC). Ultimately, the engagement of CR2 and CD4 receptors by opsonized virions leads to an increased rate of HIV spread through the tissue culture with a ten-fold increase in viral reverse transcriptase released into the culture medium and an increase in HIV genomic RNA [10]. In addition, evidence from in vitro and in vivo studies indicated that C-ADE occurs early in infection during the acute, high viremia phase $[11,12]$.

Since complement activation is an extremely potent mechanism of the innate immune system and is potentially dangerous for host cells, it is tightly regulated. This regulation is mediated by proteins such as cell surface-like membrane cofactor protein (MCP), decay accelerating factor (DAF) and protectin (CD59), and the soluble factor $\mathrm{H}(\mathrm{fH})$ that can down-regulate complement activation at several stages of cascade and protect host cells from complement-mediated damage. The complement system is strongly activated upon infection by HIV but CML of viruses is inefficient $[7,13]$. The susceptibility of HIV to CML has been shown to be dependent on the expression of the MCP and CD59 complement-regulatory proteins on infected cells [14]. Since HIV acquires the host cell membrane and its associated membrane proteins (including MCP, DAF and CD59) during budding, there is an intrinsic resistance of the virus to CML $[15,16]$. Studies have shown that blocking DAF and CD59 on HIV with specific antibodies results in an increased sensitivity of both primary isolates and laboratory-strains of HIV to CML $[17,18]$.

HIV infection results in the activation of the complement system, even in the absence of HIV-specific Abs [19] and results in the deposition of $\mathrm{C} 3$ fragments on the viral surface both in vitro [20] and in vivo [21]. HIV bound extracellularly to FDC in the germinal centers of lymph nodes represent the largest viral reservoir in HIV-infected individuals $[22,23]$. The binding of this infectious pool of HIV in the germinal centers depends mainly on interactions of CR2 expressed on FDC (or B cells) with C3d fragments attached to the viral surface $[21,24]$. In addition, an association of complement-opsonized HIV with peripheral B cells through CR2-C3d interactions has been described in HIV-infected individuals [25]. These CR2-C3d interactions between B cells and HIV are critical for efficient B cell-mediated transmission of complement-opsonized HIV to T cells [26].

\section{Complement receptor type 2 on target and bystander cells} Complement activation by the presence of HIV particles results in the generation of many $\mathrm{C} 3$-fragments that are 
recognized by different complement receptors expressed on various cell types [5]. Among these, C3dg and C3d serve as ligands for CR2 with high affinity [19]. Binding of C3d-coated particles induces a temperature-dependent aggregation of CR2 in lipid rafts on cells. The cross-linking of CR2 and the B-cell receptors through complementopsonized antigens decreases the threshold necessary for B-cell activation and contributes to a prolongation of Bcell antigen receptor signaling. Thus, CR2 plays an important role in B-cell activation and combines the innate and adaptive arms of the immune system. The CR2 on FDC can bind opsonized immune-complexes (ICs) and is important for B cell affinity maturation and the development of B-cell memory. A major mechanism of FDC trapping is binding of antibody and complement-opsonized HIV to CR2 [24,27]. Viruses retained in this way, even in the presence of neutralizing antibodies, have been shown to remain infectious in vivo for months and comprise a viral archive that can be transmitted to T cells and other target cells migrating through germinal centers. Similarly, infection of CD4+ T cells is facilitated by circulating B cells that carry HIV bound to CR2. This mode of trans-infection occurs with virus opsonized with complement alone or with complement plus antibody $[20,28]$.

\section{The hypothesis}

Recent findings have generated renewed interest in socalled "non-neutralizing" antibodies that are unable to directly inhibit free virus entry into target cells, but nonetheless, exhibit antiviral activity mediated by the Fc region of the antibody molecule. These antibody effector mechanisms include complement binding and viral lysis, phagocytosis of antibody-coated virions, and antibodydependent cellular cytotoxicity $[29,30]$. The complement system constantly interacts with HIV during all stages of infection highlighting the importance of CR2 in C-ADE. Taken together, the hypothesis presented here investigates a new strategy using a fusion protein to target and amplify complement deposition on HIV virions regardless of modulating complement inhibitor expression. The fusion protein consists of target domain, the C3-binding region of CR2, linked to a complement-activating human IgG1 Fc domain (CR2-Fc). The novel complement activator, CR2-Fc, is expected to enhance complement deposition and result in the further production of CR2 ligands through the complement-activating Fc domain. Thus, CR2-Fc will down-regulate complement inhibitors (MCP, DAF and CD59) or block their function on HIV virions that may enhance CML. More importantly, this targeted complement activator is able to bind to sites of complement activation, so it is likely to improve their efficacy while reducing potentially serious side effects resulting from complement activation. Furthermore, the human IgG1 Fc domain can also play a role of fixing complement system, so the more complement activation that occurs will lead to more CR2-Fc targeting to HIV. Subsequently, the positive feedback loop generated by the complement cascade results in enhanced lysis of HIV and preventing infection of naïve cells.

\section{Testing the hypothesis}

After preparation of human CR2-Fc fusion protein, biodistribution studies were performed to evaluate the biologic activity of CR2-Fc in vitro. HIV-1 was cultivated in H9 cells and cell-free virus obtained from supernatants. Infection experiments were performed in 24-well plates in triplicate and virus opsonization was performed by incubating appropriate dilutions of HIV in culture medium with normal human serum (NHS) and purified CR2-Fc proteins. A control group included viruses that were incubated with NHS only under the same conditions. Finally, neutralization tests were used to estimate the efficiency of CR2-Fc-enhanced lysis of HIV compared to controls.

\section{Implication of the hypothesis}

A successful test of the hypothesis would demonstrate that CR2-Fc can bind to HIV virions and can result in an amplification of the complement activation cascade. As a consequence of this action, HIV would likely be eliminated by CML and further infection by HIV should be inhibited. Furthermore, CR2-Fc bound to HIV virions is likely to reduce potential damage of host cells and tissues resulting from excess complement activation. Thus, it is meaningful to investigate the potential role of CR2-Fc for the abrogation of HIV infection in humans, as this new finding would suggest a novel approach for HIV therapy.

\section{Competing interests}

The authors declare that they have no competing interests.

\section{Authors' contributions}

YYX, CFZ, LLJ and HBS prepared the paper. CRW, HHL, YW, YSS, LYH and YSZ participated in developing the hypothesis and collaborated in writing and reviewing of the article. All authors read and approved the final manuscript.

\section{Acknowledgements}

This work was supported by the grants from the Chinese Ministry of Science and Technology 863 project (No.2007AA02ZI44) and 973 HIV research Project(No.2006CB50420I)

\section{References}

I. McCarthy M: AIDS vaccine fails in Thai trial. Lancet 2003, 362:1728.

2. McCarthy M: HIV vaccine fails in phase 3 trial. Lancet 2003 , 36I:755-756.

3. Cohen J: Promising AIDS vaccine's failure leaves field reeling. Science 2007, 3 18:28-29.

4. Walport M]: Complement. Second of two parts. N Engl J Med 2001, 344: | | 40-I| 44. 
5. Stoiber H, Banki Z, Wilflingseder D, Dierich MP: Complement-HIV interactions during all steps of viral pathogenesis. Vaccine 2008, 26:3046-3054

6. Banki Z, Stoiber H, Dierich MP: HIV and human complement: inefficient virolysis and effective adherence. Immunol Lett 2005, 97:209-2I4.

7. Stoiber H, Kacani L, Speth C, Wurzner R, Dierich MP: The supportive role of complement in HIV pathogenesis. Immunol Rev 200I, I80:168-176.

8. Robinson WE Jr, Montefiori DC, Mitchell WM: A human immunodeficiency virus type I (HIV-I) infection-enhancing factor in seropositive sera. Biochem Biophys Res Commun 1987 , 1 49:693-699.

9. Robinson WE Jr, Montefiori DC, Mitchell WM: Antibody-dependent enhancement of human immunodeficiency virus type infection. Lancet 1988, I:790-794.

10. Robinson WE: Mechanism for complement-mediated, antibody-dependent enhancement of human immunodeficiency virus type $I$ infection in MT2 cells is enhanced entry through CD4, CD2 I, and CXCR4 chemokine receptors. Viral Immunol 2006, 19:434-447.

II. Beck Z, Prohaszka Z, Fust G: Traitors of the immune systemenhancing antibodies in HIV infection: their possible implication in HIV vaccine development. Vaccine 2008, 26:3078-3085.

12. Subbramanian RA, Xu J, Toma E, Morisset R, Cohen EA, Menezes J, Ahmad A: Comparison of human immunodeficiency virus (HIV)-specific infection-enhancing and -inhibiting antibodies in AIDS patients. / Clin Microbiol 2002, 40:2|4|-2I46.

13. Sullivan BL, Knopoff EJ, Saifuddin M, Takefman DM, Saarloos MN, Sha BE, Spear GT: Susceptibility of HIV-I plasma virus to complement-mediated lysis. Evidence for a role in clearance of virus in vivo. J Immunol 1996, 157:1791-1798.

14. Saifuddin M, Ghassemi M, Patki C, Parker CJ, Spear GT: Host cell components affect the sensitivity of HIV type I to complement-mediated virolysis. AIDS Res Hum Retroviruses 1994, 10:829-837.

15. Frank I, Stoiber H, Godar S, Stockinger H, Steindl F, Katinger HW, Dierich MP: Acquisition of host cell-surface-derived molecules by HIV-I. AIDS 1996, 10:1611-1620.

16. Saifuddin M, Hedayati T, Atkinson JP, Holguin MH, Parker CJ, Spear GT: Human immunodeficiency virus type $I$ incorporates both glycosyl phosphatidylinositol-anchored CD55 and CD59 and integral membrane CD46 at levels that protect from complement-mediated destruction. J Gen Virol 1997, 78:1907-19|1.

17. Saifuddin M, Parker CJ, Peeples ME, Gorny MK, Zolla-Pazner S, Ghassemi M, Rooney IA, Atkinson JP, Spear GT: Role of virion-associated glycosylphosphatidylinositol-linked proteins CD55 and CD59 in complement resistance of cell line-derived and primary isolates of HIV-I. J Exp Med 1995, 182:50I-509.

18. Stoiber H, Pinter C, Siccardi AG, Clivio A, Dierich MP: Efficient destruction of human immunodeficiency virus in human serum by inhibiting the protective action of complement factor $\mathbf{H}$ and decay accelerating factor (DAF, CD55). Exp Med 1996, 183:307-310.

19. Stoiber H, Pruenster M, Ammann CG, Dierich MP: Complementopsonized HIV: the free rider on its way to infection. Mol Immunol 2005, 42:153-160.

20. Doepper S, Stoiber H, Kacani L, Sprinzl G, Steindl F, Prodinger WM Dierich MP: B cell-mediated infection of stimulated and unstimulated autologous T lymphocytes with HIV-I: role of complement. Immunobiology 2000, 202:293-305.

21. Banki Z, Kacani L, Rusert P, Pruenster M, Wilflingseder D, Falkensammer B, Stellbrink HJ, van Lunzen J, Trkola A, Dierich MP, Stoiber H. Complement dependent trapping of infectious HIV in human lymphoid tissues. AIDS 2005, 19:48I-486.

22. Burton GF, Keele BF, Estes JD, Thacker TC, Gartner S: Follicular dendritic cell contributions to HIV pathogenesis. Semin Immunol 2002, 1 4:275-284.

23. Smith BA, Gartner S, Liu Y, Perelson AS, Stilianakis NI, Keele BF, Kerkering TM, Ferreira-Gonzalez A, Szakal AK, Tew JG, Burton GF: Persistence of infectious HIV on follicular dendritic cells. J Immunol 200I, 166:690-696.

24. Kacani L, Prodinger WM, Sprinzl GM, Schwendinger MG, Spruth M, Stoiber H, Döpper S, Steinhuber S, Steindl F, Dierich MP: Detachment of human immunodeficiency virus type I from germi- nal centers by blocking complement receptor type 2 . J Virol 2000, 74:7997-8002

25. Moir S, Malaspina A, Li Y, Chun TW, Lowe T, Adelsberger J, Baseler M, Ehler LA, Liu S, Davey RT Jr, Mican JA, Fauci AS: B cells of HIV. I-infected patients bind virions through CD2 I-complement interactions and transmit infectious virus to activated $T$ cells. J Exp Med 2000, 192:637-646.

26. Dopper S, Wilflingseder D, Prodinger WM, Stiegler G, Speth C, Dierich MP, Stoiber H: Mechanism(s) promoting HIV-I infection of primary unstimulated $\mathrm{T}$ lymphocytes in autologous $B$ cell/T cell co-cultures. Eur J Immunol 2003, 33:2098-2 I07.

27. Ho J, Moir S, Kulik L, Malaspina A, Donoghue ET, Miller NJ, Wang W, Chun TW, Fauci AS, Holers VM: Role for CD2I in the establishment of an extracellular HIV reservoir in lymphoid tissues. Immunol 2007, I 78:6968-6974.

28. Banki Z, Wilflingseder D, Ammann CG, Pruenster M, Mullauer B, Hollander K, Meyer M, Sprinzl GM, van Lunzen J, Stellbrink HJ, Dierich MP, Stoiber H: Factor I-mediated processing of complement fragments on HIV immune complexes targets HIV to CR2expressing B cells and facilitates B cell-mediated transmission of opsonized HIV to T cells. J Immunol 2006, 177:3469-3476

29. Hessell AJ, Hangartner L, Hunter M, Havenith CE, Beurskens FJ, Bakker JM, Lanigan CM, Landucci G, Forthal DN, Parren PW, Marx PA, Burton DR: Fc receptor but not complement binding is important in antibody protection against HIV. Nature 2007, 449:101-104.

30. Willey S, Aasa-Chapman MM: Humoral immunity to HIV-I: neutralisation and antibody effector functions. Trends Microbiol 2008, 16:596-604.
Publish with BioMed Central and every scientist can read your work free of charge

"BioMed Central will be the most significant development for disseminating the results of biomedical research in our lifetime. "

Sir Paul Nurse, Cancer Research UK

Your research papers will be:

- available free of charge to the entire biomedical community

- peer reviewed and published immediately upon acceptance

- cited in PubMed and archived on PubMed Central

- yours - you keep the copyright
BioMedcentral 